\title{
Procedimientos estadísticos utilizados en las tesis de maestría en Agricultura Tropical Universidad de Oriente Periodo 1998-2016
}

\author{
Statistical procedures used in the works of master in tropical \\ agriculture Universidad de Oriente Period 1998-2016 \\ Guillermo Romero-Marcano ${ }^{\mathrm{a}}$ \\ guillermo.ro80@gmail.com \\ Ramon Silva-Acuña ${ }^{b}$ \\ drramonsilvaa@gmail.com \\ Renny Barrios-Maestre ${ }^{\mathrm{c}}$ \\ rennybarrios@gmail.com
}

\begin{abstract}
Resumen
Con el objetivo de estudiar los procedimientos estadísticos utilizados en el postgrado en Agricultura Tropical (PAT) de la Universidad de Oriente, periodo 1998-2016, se analizó el manejo estadístico aplicado en los 53 trabajos de maestría presentados, identificando variables relacionadas con el diseño experimental, arreglo de tratamientos, número de repeticiones, características de la unidad experimental, tipos de variables y escalas utilizadas, tipo de análisis estadísticos, verificación de supuestos requeridos por las pruebas e interpretación de interacciones, entre otros. La información fue sistematizada en una base de datos y analizada a través de estadística descriptiva, prueba binomial y análisis de correlación, según la variable analizada. Se determinó que el uso de técnicas estadísticas difiere entre las menciones del PAT. La tendencia es hacia el desarrollo de experimentos críticos con empleo de diseños experimentales y arreglos de tratamientos robustos. Más de 60 por ciento de los experimentos emplean menos de cuatro repeticiones y hay deficiencias en la descripción de las unidades experimentales. Los supuestos del ANAVA no son verificados y hubo deficiencias en la interpretación de interacciones significativas. Las pruebas de comparación de promedios más frecuentes fueron Duncan y Mínima Diferencia Significativa (42,42 y 30,30 por ciento, respectivamente), las cuales inducen error tipo I.
\end{abstract}

Palabras clave: Diseño experimental, comparación de promedios, interacción, ANAVA.

\footnotetext{
${ }^{a}$ Departamento de Nutrición Animal y Forrajes, Escuela de Zootecnia, Universidad de Oriente, Núcleo Monagas

b Área de Diseño de experimentos y Análisis estadístico, Postgrado de Agricultura Tropical, Universidad de Oriente, Núcleo Monagas

${ }^{\mathrm{c}}$ Instituto Nacional de Investigaciones Agrícolas, San Agustín de la Pica, Monagas, Venezuela
} 


\begin{abstract}
With the aim of studying the statistical procedures used in the postgraduate course in Tropical Agriculture (PAT) of the Universidad de Oriente, period 1998-2016, the statistical management applied in 53 master work presented was analyzed. Variables related to the experimental design, treatment arrangement, number of repetitions, characteristics of the experimental unit, types of variables and scales used, type of statistical analysis, verification of assumptions required by the tests and interpretation of interactions, among others, were identified. The information was systematized in a database and analyzed through descriptive statistics, Binomial test and correlation analysis, according to the variable analyzed. It was determined that the use of statistical techniques differs between mentions of the PAT. The tendency is towards the development of critical experiments with the use of robust experimental designs and treatment arrangements. More than 60 percent of the experiments employ less than four repetitions and there are deficiencies in the description of the experimental units. The assumptions of the ANAVA are not verified and there were deficiencies in the interpretation of significant interactions. The most frequent comparison tests of media were Duncan and Minimum Difference Significant (42.42 and 30.30 percent, respectively), which induce type I error.
\end{abstract}

Keywords: Experimental design, comparison of media, interaction, ANAVA.

\title{
1. Introducción
}

En el desarrollo de la ciencia en general y en particular las ciencias biológicas, el conocimiento de la metodología estadística es una herramienta imprescindible para la obtención, análisis e interpretación de datos que proceden de observaciones sistemáticas o de investigación, donde es necesario conocer los efectos de uno o varios factores que intervienen en los fenómenos objetos de estudio (Badii et al. 2007). La estadística es esencial para la investigación agrícola, con grandes aportes a la agronomía moderna en áreas como: fertilización, control de plagas y enfermedades, entre otras (Jiménez y Reyes 2013); por ello, es disciplina obligatoria en los programas formativos para agrotécnicos (Angarita y Parra 2014).

El programa de estudios de Postgrado de la Universidad de Oriente, Núcleo Monagas, se creó en 1978, con estudios de cuarto nivel en Agricultura Tropical, en las menciones: Botánica Agrícola y Fisiología Vegetal; posteriormente en 1994, se incorporan las menciones Edafología, Mejoramiento de Plantas y Producción Vegetal(UDO 2015). Desde sus inicios, esta maestría representó una alternativa válida y de calidad para formación de investigadores en el área agrícola (Alegría 1998).

Entre las principales reseñas históricas referentes a revisiones oficiales de procedimientos estadísticos se encuentra la realizada por el matemático y estadístico Víctor Chew(1976)a mediados de los años 60, quien a solicitud del Servicio de 
Investigación Agrícola de los Estados Unidos revisó el documento ARS 20-3, que hasta el momento representaba la referencia en el procesamiento estadístico de promedios, mediante el uso de análisis de varianza (ANAVA) y pruebas de comparaciones múltiples. A partir de dicha revisión se establecieron nuevos criterios de análisis estadístico para disminuir sensiblemente errores tipo I y tipo II generados por las pruebas de medias (ARS 1977).

Posteriormente, Petersen (1977) evaluó el volumen 68 de la Revista Agronomy Journal, en relación a la aplicación de pruebas de comparaciones múltiples y recomendó su utilización únicamente en datos provenientes de experimentos con tratamientos cualitativos no estructurados. Por otro lado, Johnson y Berger (1982) y Maddenet al.(1982),revisaron los volúmenes 70 y 67 de la revista Phytopathology, respectivamente, y cuestionaron el uso de pruebas de comparaciones múltiples en investigaciones fitopatológicas, señalando que más del 60 por ciento de los artículos revisados usaron de forma inapropiada estos procedimientos. De manera similar, Maindonald y Cox (1984) analizaron el uso de la estadística en dos revistas orientadas a agricultura (DSIR Agricultural Journals) y reportaron que los procedimientos estadísticos fueron utilizados mecánicamente, con problemas como: aplicación de pruebas de comparación múltiples a datos estructurados y desinformación en los detalles del diseño experimental utilizado.

Martínez y Galindo (1994) realizaron una revisión sobre los diseños experimentales y métodos estadísticos utilizados en artículos de seis revistas científicas internacionales para el período 1990-1992 y concluyeron que los diseños experimentales más usados son el DCA y DBA, la prueba de comparación múltiple más empleada es la de Mínima diferencia significativa (MDS); mientras que el uso de técnicas multivariadas es reducido y se limita al campo de genética y mejoramiento vegetal. Por su parte, Silva-Acuña et al. (2000) exponen la necesidad de emplear procedimientos de contrastes ortogonales al realizar comparaciones de medias de tratamientos cualitativos estructurados.

En otros ámbitos, particularmente en el caso de la psicología y la medicina, Pardo et al. (2007), detectaron errores en la interpretación de la interacción significativa de factores; Zinsmeister y Connor (2008) señalan que los errores más comunes consistieron en no definir la metodología utilizada y el análisis apropiado, incongruencia entre objetivos y conclusiones; generar conclusiones sin soporte de datos; uso de diferencias dentro de un grupo para concluir diferencias entre grupos; análisis de variables correlacionadas como variables independientes e incorrecta interpretación del valor de la probabilidad. De manera análoga, Romani et al. (2010) indican que en revistas peruanas de medicina, el manejo estadístico más utilizado en los artículos corresponde a estadística descriptiva (83 por ciento), prueba t Student (11 por ciento) y pruebas no paramétricas (9 por ciento); sin embargo, la frecuencia de uso de estadística descriptiva fue descendiendo durante el periodo evaluado. De manera similar, Thiese et al. (2015) concluyeron que ocurre aplicación incorrecta de pruebas estadísticas, creación de modelos multivariados incompletos o incorrectos y exclusión de valores atípicos en las pruebas estadísticas utilizadas en investigación biomédica. 
En el área de docencia, Echavarría et al. (2006) al evaluar trabajos de grado de la Facultad de Ciencias Agropecuarias de la Universidad Nacional (Colombia) durante el periodo 1999-2001, determinaron que casi la mitad de los trabajos presentaron al menos un error, entre los cuales destacan: aleatorización deficiente o nula, incumplimiento de los supuestos del ANAVA, uso de pruebas de medias forzadas e interpretación inadecuada de la interacción en experimentos factoriales. Por su parte, Lizarzaburu (2013) analizando 36 trabajos de investigación seleccionados aleatoriamente de la Universidad San Pedro, Perú, durante el periodo 2009-2011, concluye que el 89 por ciento de los trabajos utiliza técnicas estadísticas, siendo la estadística descriptiva la más utilizada; solo 11 por ciento de los trabajos prueban hipótesis correctamente y apenas el 6 por ciento de los trabajos analizan los supuestos requeridos para la aplicación de las pruebas.

En función de lo anteriormente planteado la presente investigación se realizó con el objetivo de revisar y analizar los procedimientos estadísticos utilizados en la elaboración de las tesis de maestría del Postgrado de Agricultura Tropical de la Universidad de Oriente, durante el periodo 1998-2016.

\section{Metodología}

El presente estudio se realizó bajo la metodología de investigación documental, del tipo descriptivo a partir de la información disponible en la biblioteca física del Postgrado en Agricultura Tropical, Campus Juanico, Universidad de Oriente, Núcleo Monagas. Se revisaron todos los trabajos de maestría presentados de manera oficial entre los años 1998 y 2016, 53 ejemplares en total, correspondientes a las cinco menciones impartidas, distribuidas de la siguiente manera: 2 en Mejoramiento de Plantas, 3 en Botánica Agrícola, 10 en Fisiología Vegetal, 20 en Edafología y 18 en Producción Vegetal.

Se procedió a desglosar el manejo estadístico aplicado en el procesamiento de los resultados de cada trabajo de maestría, identificando variables relacionadas con el diseño experimental utilizado, el arreglo de los tratamientos, el número de repeticiones, características de la unidad experimental, tipo de variables evaluadas, escalas de medición, tipo de análisis estadísticos aplicados, cumplimiento de supuestos relacionados al análisis e interpretación de interacciones, entre otros. La sistematización de la información se realizó a partir de una base de datos elaborada en el software ofimático MS-Excel 2010, a partir de la cual se determinaron las frecuencias relativas de las categorías de las respuestas.

Por mención, se calculó la frecuencia relativa del tipo de estadística utilizada, para lo cual se consideró el criterio de clasificación descrito por Arriaza-Balmón (2006), el cual separa los tratamientos estadísticos en: Análisis univariante o descriptivo (medidas de tendencia central, dispersión, distribución de frecuencias, simetría y curtosis); Análisis bivariante: pruebas paramétricas (prueba de t, correlación de Pearson, análisis de varianza para un factor), pruebas no paramétricas (MannWhitney, correlación de Spearman, Kruskal Wallis, cuadros de contingencia de chi 
cuadrado); Análisis multivariante: Análisis de varianza a partir de dos factores, análisis multivariante de la varianza (MANOVA), análisis discriminante, regresión lineal múltiple, regresión logística, covarianza, modelo lineal general (GLM), análisis de componentes principales y análisis de conglomerados.

Se cuantificó la frecuencia relativa de los distintos diseños experimentales utilizados en los trabajos de grado, así como la de los análisis estadísticos aplicados en cada estudio, incluyendo las pruebas de comparaciones múltiples y las técnicas de presentación de resultados. Las diferencias de proporciones, en la aplicación de los diseños experimentales y los arreglos de tratamientos, fueron analizadas por prueba binomialal 5 por ciento de probabilidad.

De manera similar, se realizó la cuantificación y análisis de los ítems: número de repeticiones por experimento, tipo y tamaño de la unidad experimental (UE), tipo y escala de medida de la variable independiente, tipo, escala de medida y coeficiente de variación de la variable dependiente; supuesto de normalidad; supuesto de homogeneidad; supuesto de independencia; nivel de significancia; interpretación de la interacción significativa; presentación de valores por UE en el apéndice y resumen del ANAVA en la discusión.

Se aplicó análisis de correlación de Spearman, para determinar asociación entre los ítems, factores (variables independientes) y variables dependientes del trabajo de maestría en función del año de publicación del mismo; así como también entre el tamaño de la unidad experimental y el coeficiente de variación. Todos los procedimientos estadísticos fueron realizados en el programa Infostat@ versión 2017 (Di Rienzo et al. 2017).

\section{Resultados y discusión}

\section{Estadística utilizada}

La gran mayoría de los trabajos de maestría presentados durante el periodo 19982016 en el Postgrado en Agricultura Tropical de la Universidad de Oriente, aplican procedimientos estadísticos, con excepción de la mención de Edafología donde sólo el 65 por ciento usa la herramienta estadística y el 35 por ciento restante aplica enfoque documental (Figura 1). En investigación científica relacionada con agricultura, la estadística es base esencial (Jiménez y Reyes 2013) de allí que los estudios biométricos combinen, la medición de aspectos biológicos con el desarrollo y aplicación de métodos estadísticos, para extraer información a partir de datos cuantitativos y cualitativos (Balzarini et al. 2013).

A pesar que la estadística constituye una herramienta sumamente útil y poderosa, de amplia utilización en la realización de investigación y obviamente en la redacción sistemática de artículos científicos (Díaz y Rivas 2015), existen trabajos de investigación que pueden salirse del patrón, como fue el caso de Mendel (1865) en sus experiencias de hibridación en plantas o el de Watson y Crick (1953) al estudiar la estructura molecular del ADN, entre otros. Actualmente ese enfoque 
resulta de cierta forma ambiguo debido a la necesidad imperativa de demostrar fehacientemente los hallazgos reportados, lo cual involucra discusiones estadísticas de investigaciones previas.

En el caso particular de la mención Edafología, el enfoque documental de los trabajos de maestría están basados en la aplicación de esquemas para la evaluación de tierras y en propuestas teóricas para el establecimiento de sistemas de producción sustentables, los cuales no ameritaron el uso de estadística para interpretar los fenómenos abordados, según el criterio de los autores.

En lo que respecta al tipo de estadística aplicado en los trabajos de maestría, se constató tendencias distintas de acuerdo a la mención: en Mejoramiento de Plantas el 100 por ciento de los trabajos se realizó bajo análisis multivariante; en Botánica Agrícola el 66,67 por ciento por análisis univariante y el 33,33 por ciento restante por análisis multivariante; mientras que en los trabajos de las menciones Fisiología Vegetal y Producción Vegetal resultó más frecuente la aplicación de análisis multivariante y bivariante y en menor frecuencia el análisis univariante, y en la mención de Edafología predominó el análisis bivariante (Figura 1).

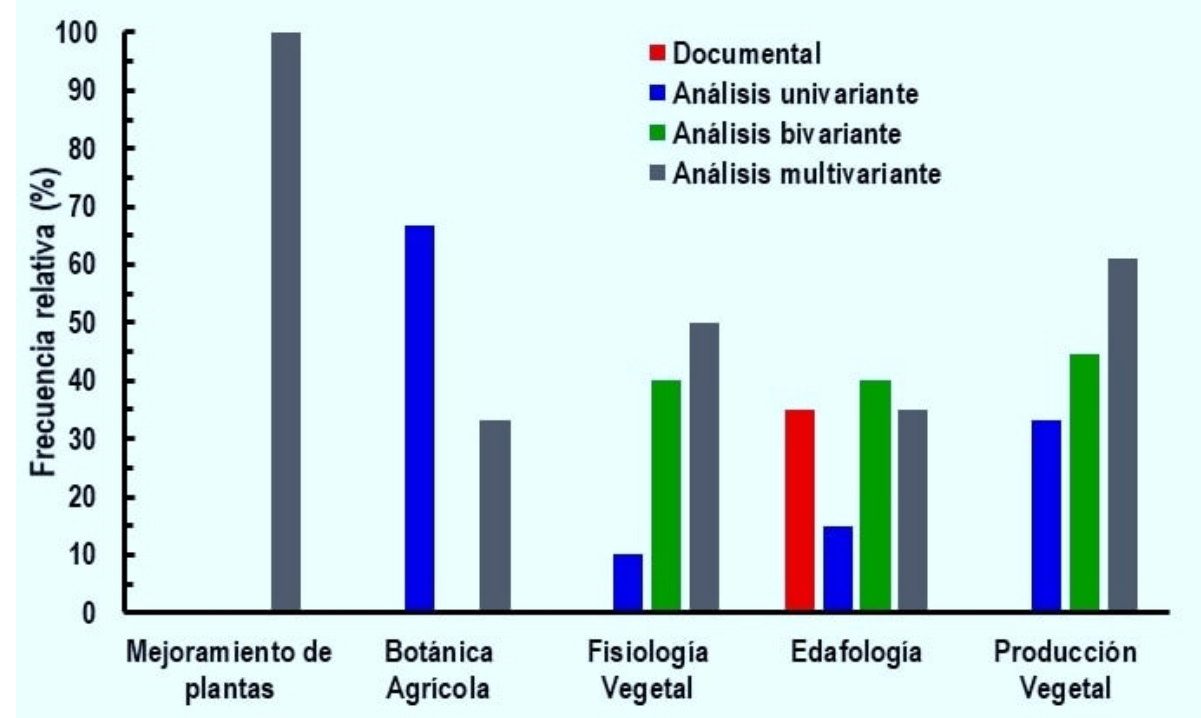

Figura 1. Tipos de investigación y tipo de estadística utilizada en los trabajos de grado del postgrado en Agricultura Tropical distinguidos por mención.

El mejoramiento de plantas busca entender la compleja interacción genotipo ? ambiente e identificar cultivares de alto rendimiento (Yan et al. 2001), a través de métodos estadísticos apropiados (Estrada y Martínez 2003); siendo los análisis multivariantes, los más eficientes, al permitir enfrentar diferentes variables o factores independientes, juntos (asociados o no a covariables) con una o más variables dependientes (Johnson 2000). Por el contrario, la botánica, asociada a taxonomía y sistemática vegetal, incluye el estudio, clasificación y nomenclatura de las plantas, así como, las evidencias científicas que las sustentan (Catalán 2016), y a través 
de la estadística descriptiva pueden resumirse tales evidencias de manera sencilla y clara para su interpretación, utilizando como herramientas cuadros, figuras e imágenes (Rendón-Macías et al. 2016).

En los trabajos de maestría de las menciones Fisiología Vegetal, Edafología y Producción Vegetal predominaron los análisis bivariantes y multivariantes a través de vías paramétricas, lo que evidencia la necesidad de transcender del ámbito descriptivo al plano inferencial (Sabadías 1995), al incorporar más variables (independientes y/o dependientes) y estudiar la relación directa entre ellas (Tabachnick y Fidell 2001).

Diseños experimentales y análisis estadísticos

En la Tabla 1 se observan los valores para las variables diseños experimentales y arreglos de tratamientos aplicados en los trabajos de maestría revisados. El análisis estadístico detectó efecto significativo entre las proporciones de aplicación de los diseños experimentales, siendo el diseño en bloques al azar (DBA) el más aplicado, en comparación al diseño completamente aleatorizado (DCA); es destacable que ningún trabajo revisado consideró la aplicación del diseño cuadrado latino (DCL). Entre los arreglos de tratamientos aplicados, el análisis estadístico también detectó efecto significativo, siendo el arreglo factorial el más aplicado, en comparación a los arreglos en parcelas, mientras que, las diferencias de proporciones de aplicación entre arreglos en parcelas (divididas y subdivididas), no fueron significativas; ninguno de los trabajos de maestría revisados aplicó arreglo en parcelas subsubdivididas.

El diseño de bloques al azar está entre los más frecuentemente utilizados en experimentación agrícola (Martínez y Galindo 1994; Ruiz-Ramírez et al. 2012); las virtudes que presenta, son: reducción de la varianza de error; facilidad de análisis; flexibilidad y precisión, siendo 67 por ciento más eficiente que el DCA (Baddi et al. 2007). Este diseño se presta para comparar una gama enorme de materiales: animales, plantas, tratamientos químicos, entre otros y puede construirse tanto en campo como en laboratorio, generalmente se aplica cuando el factor causante de variabilidad adicional, es conocido y controlable, para eliminar su efecto en la comparación estadística de los tratamientos (Monzón 1992).

A pesar de que los cuadrados latinos presentan gran variedad de aplicaciones en el área de las matemáticas (Cortés 2011) se hace poco frecuente en investigación agrícola (Echavarría et al. 2006), posiblemente debido a las condiciones limitativas que este diseño obliga: igual número de niveles por factor, cada tratamiento aparece una vez por fila y por columna y número fijo de unidades experimentales, es decir, número de tratamientos al cuadrado (Monzón 1992).

Tabla 1. Diseños experimentales y arreglos de tratamientos aplicados en los trabajos de maestría del postgrado en Agricultura Tropical, durante el periodo 19982016. 


\begin{tabular}{|c|c|c|c|c|c|}
\hline \multirow{2}{*}{\multicolumn{2}{|c|}{$\begin{array}{l}\text { Diseño experi- } \\
\text { mental }\end{array}$}} & \multicolumn{2}{|c|}{ Aplicación } & \multicolumn{2}{|c|}{$\begin{array}{l}\text { Diferencia de propor- } \\
\text { ciones } \\
(\text { Valor } p \text { ) }\end{array}$} \\
\hline & & Si & No & & \\
\hline \multicolumn{2}{|c|}{$\begin{array}{l}\text { Diseño Comple- } \\
\text { tamente Aleato- } \\
\text { rizado }\end{array}$} & 10 & 23 & \multicolumn{2}{|l|}{$\begin{array}{l}-0,4848 \\
(<0,0001)\end{array}$} \\
\hline \multicolumn{2}{|c|}{$\begin{array}{l}\text { Diseño Bloques } \\
\text { al Azar }\end{array}$} & 26 & 7 & & \\
\hline \multicolumn{2}{|c|}{$\begin{array}{l}\text { Diseño Cuadra- } \\
\text { do Latino }\end{array}$} & 0 & 0 & \multicolumn{2}{|l|}{$\mathrm{N} / \mathrm{A}$} \\
\hline \multicolumn{6}{|c|}{$\begin{array}{l}\text { Arreglo de } \\
\text { tratamientos }\end{array}$} \\
\hline \multicolumn{2}{|c|}{ Arreglo factorial } & 27 & 6 & $\begin{array}{l}0,4848 \\
(<0,0001)\end{array}$ & $\mathrm{N} / \mathrm{A}$ \\
\hline \multirow[t]{3}{*}{$\begin{array}{l}\text { Arreglo en } \\
\text { Parcelas } \\
\end{array}$} & Divididas & 6 & 27 & & $\begin{array}{l}0,0303 \\
(0,7553)\end{array}$ \\
\hline & Subdivididas & 5 & 28 & & \\
\hline & Subsubdivididas & 0 & 0 & $\mathrm{~N} / \mathrm{A}$ & \\
\hline
\end{tabular}

La mayor frecuencia en el uso de arreglos factoriales, corresponde con la tendencia antes descrita, vinculada a la necesidad de estudiar varios factores por experimento, con la ventaja de poder considerar adicionalmente, con estos modelos, el efecto de interacción (Montgomery 2003); además de estudiar la interacción, los arreglos factoriales permiten ahorrar recursos e incrementar la precisión de estimación (Sahagún-Castellanos et al. 2008) con eficiencia de hasta 188 por ciento por encima de los experimentos de un solo factor (Baddi et al. 2007).

En la Tabla2, se presentan los análisis estadísticos aplicados de acuerdo a la condición experimental, se puede observar que el análisis estadístico más frecuente fue el análisis de varianza de dos o más factores (ANAVA II), relacionado directamente con las frecuencias de uso del DBA y el arreglo factorial, antes descritas; mientras que, los menos frecuentes fueron los modelos de crecimiento y el análisis de medidas repetidas. En estudios donde no se empleó algún diseño experimental, el análisis estadístico más frecuente fue la estadística descriptiva con 69,23 por ciento de los casos. Se debe resaltar que 23,08 por ciento de los trabajos bajo esta condición experimental aplicaron análisis de varianza de un factor (ANAVA I), donde cabe la posibilidad de que el investigador haya propuesto realizar un diseño experimental, pero este no fue especificado dentro de la metodología, siendo obviado en la redacción del escrito.

Tabla 2. Análisis estadísticos aplicados en los trabajos de maestría del postgrado en Agricultura Tropical, durante el periodo 1998-2016, según condición experimental. 
Procedimientos estadísticos utilizados en las tesis de maestría en Agricultura Tropical Universidad de Oriente Periodo 1998-20165

\begin{tabular}{|l|l|l|l|}
\hline Condición & Análisis estadístico & $\mathbf{N}$ & $\mathbf{\%}$ \\
\hline $\begin{array}{l}\text { Con diseño experimen- } \\
\text { tal }\end{array}$ & ANAVA I & 6 & 18,18 \\
\hline & ANAVA II & 27 & $\mathbf{8 1 , 8 2}$ \\
\hline & Análisis de regresión & 6 & 18,18 \\
\hline & Kruskal Wallis & 2 & 6,06 \\
\hline & Modelos de crecimiento & 1 & 3,03 \\
\hline & $\begin{array}{l}\text { Análisis medidas repe- } \\
\text { tidas }\end{array}$ & 1 & 3,03 \\
\hline $\begin{array}{l}\text { Sin diseño experimen- } \\
\text { tal }\end{array}$ & Estadística descriptiva & 9 & $\mathbf{6 9 , 2 3}$ \\
\hline & Prueba Kruskal Wallis & 2 & 15,38 \\
\hline & Prueba de Friedman & 1 & 7,69 \\
\hline & $\begin{array}{l}\text { Análisis de conglome- } \\
\text { rados }\end{array}$ & 2 & 15,38 \\
\hline & ANAVA I & 3 & 23,08 \\
\hline
\end{tabular}

La investigación cuantitativa se clasifica en: Experimental (que involucra el experimento propiamente dicho y aplica aleatorización, manipulación de variables y control rígido, para establecer relaciones de causa-efecto) y no experimental (basado en muestreo y observación, con la intención de describir, diferenciar o examinar asociaciones) (Sousa et al. 2007; Montoya-Márquez et al. 2011). Según el objeto de investigación, existen pruebas estadísticas específicas (Boubeta y Mallou 2014), donde para las estructuras básicas del diseño experimental (DCA, DBA y DCL), son apropiados los modelos ANAVA, siempre que los datos se distribuyan normalmente (Kirk 1995), mientras que, en investigación por muestreo, la estadística descriptiva es la que mejor se ajusta (Sousa et al. 2007).

El análisis de varianza (ANAVA), como cualquier otro procedimiento estadístico paramétrico, requiere que los datos a analizar cumplan los supuestos previos de normalidad, homogeneidad e independencia de los errores (Dawson-Saunders y Trapp 1993), para ello se necesitan datos producidos bajo diseños experimentales que apliquen correctamente los principios de replicación, aleatorización y control local (Montoya-Márquez et al. 2011); en tal sentido, se debe considerar incorrecta la aplicación del ANAVA, en datos generados por muestreo u observación, debido al riesgo de cometer errores de interpretación (Tipo I y II) y generar conclusiones poco válidas (Badii et al. 2007).

Las pruebas de comparación de medias utilizadas en los trabajos de maestría revisados se muestran en la Tabla 3, siendo las más frecuentes la prueba de rangos múltiples de Duncan y la prueba de mínima diferencia significativa (MDS). También resalta el uso de la Prueba de Bayes en 15,15 por ciento de los trabajos revisados.

Tabla 3. Pruebas de comparación de medias utilizadas en los trabajos de maestría del Postgrado en Agricultura Tropical, durante el periodo 1998-2016. 


\begin{tabular}{|l|l|l|}
\hline $\begin{array}{l}\text { Prueba de comparación de } \\
\text { medias }\end{array}$ & $\mathbf{n}$ & $\mathbf{\%}$ \\
\hline Bayes & 5 & 15,15 \\
\hline $\begin{array}{l}\text { Comparación de grupos de Fried- } \\
\text { man }\end{array}$ & 1 & 3,03 \\
\hline $\begin{array}{l}\text { Mínima diferencia significativa } \\
\text { (MDS) }\end{array}$ & 10 & 30,30 \\
\hline Rangos múltiples de Duncan & 14 & 42,42 \\
\hline Tukey HSD & 3 & 9,09 \\
\hline
\end{tabular}

Las pruebas de media o pruebas post hoc, se realizan únicamente después que el ANAVA ha generado un valor de F significativo (Blanco 2001). Su utilidad ha sido fuertemente cuestionada por la literatura, debido a inconsistencias de aplicabilidad e interpretación, siendo catalogadas como fantasías estadísticas y nidos de culebra (Nelson y Rawlings 1983; Mihail y Niblack 1991).

A pesar de su alta frecuencia de uso, contradictoriamente, las pruebas de rangos múltiples de Duncan y de MDS son las menos recomendadas por los estadísticos, debido a que rechazan la hipótesis nula con facilidad, y diferencias muy pequeñas son declaradas significativas de manera incorrecta (Carmer y Swanson 1973; Maindonald y Cox 1984; Dawson-Saunders y Trapp 1993). Las más recomendadas son las pruebas de Scheffe, Tukey HSD y Student-Newman-Keuls (SNK), debido a su alta potencia para detectar diferencias de forma correcta y evadir errores de interpretación (Tipo I) (Madden et al. 1982). Otras pruebas como: Mínima diferencia significativa de Fisher (FLSD), Mínima diferencia significativa de Bayes (BLSD) y análisis de conglomerados Scott Knott, resultan aún más competentes, debido a sus altas tasas de decisión correcta (Carmer y Swanson 1973).

Número de repeticiones

En la Tabla 4 se presenta el número de repeticiones utilizadas por experimento en los trabajos de maestría revisados, las mayores frecuencias corresponden a experimentos con cuatro y tres repeticiones $(41,30$ y 36,96 por ciento respectivamente). Destaca la existencia en los trabajos revisados de experimentos con una sola observación y hasta con veinte repeticiones.

Para experimentos agronómicos, el número óptimo de repeticiones esta entre 4 y 6 , pero en algunos casos es necesario aplicar por encima de 8 repeticiones (Raudonius 2017). El número mínimo de repeticiones por experimento puede calcularse con base a los grados de libertad asignados al error experimental, esperando mínimo 10 grados. Bajo este principio, el número mínimo de tratamientos y de repeticiones por experimento serian: 3-6; 4-5; 5-4; 6-3; 7-3; 8-3, respectivamente (Monzón 1992).

Tabla 4. Número de repeticiones utilizadas por experimento en los trabajos de maestría del Postgrado en Agricultura Tropical, durante el periodo 1998-2016. 


\begin{tabular}{|l|l|l|}
\hline $\begin{array}{l}\text { Número } \\
\text { Repeticiones }\end{array}$ & $\mathbf{n}$ & $\mathbf{\%}$ \\
\hline 1 & 1 & 2,17 \\
\hline 3 & 17 & $\mathbf{3 6 , 9 6}$ \\
\hline 4 & 19 & $\mathbf{4 1 , 3 0}$ \\
\hline 5 & 6 & 13,04 \\
\hline 6 & 1 & 2,17 \\
\hline 7 & 1 & 2,17 \\
\hline 9 & 1 & 2,17 \\
\hline 20 & 1 & 2,17 \\
\hline
\end{tabular}

Las repeticiones permiten: estimar el error experimental, mejorar la precisión del experimento al reducir el error estándar; aumentar el alcance de la inferencia del experimento y ejercer control sobre la varianza del error(Fernández et al. 2010). Un error muy frecuente es confundir las mediciones repetidas realizadas sobre una misma unidad experimental como repeticiones, denominadas pseudorepeticiones, que provocan una falsa creencia de precisión en el experimento, a merced únicamente del error de muestreo (Balzarini et al. 2013).

\section{Unidad experimental}

Las características de la unidad experimental utilizada por experimento se muestran en la Tabla 5, se observa que 39,13 por ciento de los trabajos no describen la unidad experimental utilizada. En los trabajos revisados, el tamaño de la unidad experimental está representado por recipientes, bolsas de vivero de $1 \mathrm{~kg}$, capsulas de Petri, hojas, suelo, agua y por algunas plantas, que corresponde a una observación. Las unidades experimentales representadas por bandejas de germinación, semillas, frutos y plantas, en su mayoría abarcan un tamaño entre 3 y 200 observaciones.

Tabla 5. Unidad experimental utilizada por experimento en los trabajos de maestría del postgrado en Agricultura Tropical, durante el periodo 1998-2016,

\begin{tabular}{|c|c|c|c|}
\hline Tipo de objeto & $\begin{array}{l}\text { Tamaño de la uni- } \\
\text { dad experimental }\end{array}$ & $\mathbf{n}$ & $\%$ \\
\hline \multicolumn{2}{|l|}{ Sin descripción } & 18 & 39,13 \\
\hline Bandejas de germinación & 40 y 200 alveolos & 2 & 4,35 \\
\hline $\begin{array}{l}\text { Recipiente, bolsa de } 1 \mathrm{~kg} \text {, } \\
\text { compostero }\end{array}$ & 1 unidad & 3 & 6,52 \\
\hline Semillas & $\begin{array}{l}10 \text { a } 50 \text { semillas; } 10 \text { a } 25 \\
\text { esquejes; } 6 \text { explantes }\end{array}$ & 6 & 13,04 \\
\hline Frutos & 5 a 48 frutos & 4 & 8,70 \\
\hline Plantas & $1,3,5,8$ y 10 plantas & 6 & 13,04 \\
\hline Capsulas de Petri & 1 capsula & 3 & 6,52 \\
\hline Hoja o foliolo & 1 unidad & 2 & 4,35 \\
\hline Suelo o agua & $\begin{array}{l}1 \text { muestra compuesta; } \\
10 \mathrm{~kg} \text { de suelo }\end{array}$ & 10 & 21,74 \\
\hline
\end{tabular}

Comunicaciones en Estadística, junio 2020, Vol. 13, No. 1 
Las características de la unidad experimental, en cualquier estudio, determinan la validez externa (representatividad con lo real) e interna (comparabilidad entre grupos) y regulan el error experimental (Baray 2006). Unidades experimentales deficientes en cantidad y calidad, provocan disminución de la potencia estadística para detectar efectos de tratamientos y detección de diferencias estadísticas confundidas, debido a otros factores ajenos al interés del investigador (MontoyaMárquez et al. 2011).

$\mathrm{Al}$ igual que para las repeticiones, cada unidad experimental debe ser realmente independiente para evitar alterar los supuestos de parametricidad (Balzarini et al. 2013); es típico confundir los términos unidad experimental y observación, la diferencia radica en que la unidad experimental es a quien se aplica el tratamiento independientemente y puede estar compuesta por una o varias unidades de observaciones; la varianza entre las observaciones múltiples de la misma unidad experimental, es una medida equivocada de error experimental (Kuehl 2001), siendo equiparable al efecto producido por las pseudorepeticiones.

Tipos de variables

En la Tabla 6, se presentan los tipos de variable independiente utilizados en los trabajos de maestría revisados, donde se observa mayor frecuencia de variables cualitativas o categóricas (58,14 por ciento) en comparación a las variables cuantitativas (41,86 por ciento); dentro de las cuales, el 98,66 por ciento está representado por variables de tipo nominal, mientras que, en las variables cuantitativas el 100 por ciento resultó de tipo de intervalo.

En cualquier experimento, el tipo de variable que exprese el factor tratamiento, estará acorde con el objetivo del investigador, el análisis estadístico más conveniente (Fuentes 2015). Para tratamientos cualitativos no estructurados, tipo nominal, se recomienda determinar diferencias significativas mediante ANAVA y pruebas de comparaciones múltiples; tratamientos cualitativos estructurados del tipo ordinales), deben analizarse por contrastes ortogonales, mientras que tratamientos cuantitativos (de intervalo o de razón), recomiendan ser procesados mediante análisis de regresión (Blanco 2001).

Tabla 6. Tipo de variables utilizadas en los trabajos de maestría del Postgrado en Agricultura Tropical, durante el periodo 1998-2016.

\begin{tabular}{|l|l|l|l|l|}
\hline $\begin{array}{l}\text { Tipo de va- } \\
\text { riable }\end{array}$ & \multicolumn{2}{|l|}{$\begin{array}{l}\text { Variable inde- } \\
\text { pendiente }\end{array}$} & $\begin{array}{l}\text { Variable } \\
\text { diente }\end{array}$ & depen- \\
\hline & $\mathbf{n}$ & $\mathbf{\%}$ & $\mathbf{N}$ & $\mathbf{\%}$ \\
\hline Cualitativa & 75 & $\mathbf{5 8 , 1 4}$ & 92 & 8,04 \\
\hline Nominal & 74 & $\mathbf{9 8 , 6 6}$ & 91 & $\mathbf{9 8 , 9 1}$ \\
\hline Ordinal & 1 & 1,34 & 1 & 1,09 \\
\hline Cuantitativa & 54 & 41,86 & 1052 & $\mathbf{9 1 , 9 6}$ \\
\hline Ordinal & 0 & $\mathbf{0}$ & 91 & 8,65 \\
\hline Intervalo & 54 & $\mathbf{1 0 0}$ & 868 & $\mathbf{8 2 , 5 1}$ \\
\hline Razón & 0 & 0 & 93 & 8,84 \\
\hline
\end{tabular}

Comunicaciones en Estadística, junio 2020, Vol. 13, No. 1 
El análisis estadístico detectó correlación negativa altamente significativa entre el número de factores (variables independientes) y el año de publicación del trabajo de maestría, lo que evidencia que a medida que avanza el tiempo en el Postgrado de Agricultura Tropical se consideran menor número de factores (variables independientes) en los experimentos, reflejando la evolución de investigaciones del tipo preliminar, donde se comparan un gran número de tratamientos, hacia el desarrollo de experimentos cada vez más críticos y específicos.

En lo que respecta al tipo de variable dependiente utilizada en los trabajos de maestría, se obtuvo mayor frecuencia para variables cuantitativas (91,96 por ciento) en comparación con las de tipo cualitativa (8,04 por ciento). En las cuantitativas, el 82,51 por ciento está representado por variables de intervalo; mientras que en las cualitativas, el 98,91 por ciento corresponde a variables nominales (Cuadro 6). Estos valores sustentan el comportamiento observado previamente en el uso de las distintas pruebas estadísticas, donde el ANAVA I y II y la estadística descriptiva fueron las más utilizadas.

Así como para cada tipo de variable independiente (tratamiento) existe un análisis estadístico conveniente, cada tipo de variable dependiente (respuesta) tiene el análisis estadístico más recomendable (Orlandoni 2010). Para variables cualitativas (nominales y ordinales) las pruebas de comparación más convenientes son los análisis no paramétricos (Chi cuadrado, Mann-Whitney, Kruskal-Wallis, Friedman, entre otros); mientras que para variables cuantitativas (ordinales, intervalo o de razón), según el cumplimiento de los supuestos, será más conveniente el uso de pruebas paramétricas (Prueba t Student, ANAVA I, ANAVA II, correlación, regresión, entre otros) (Gómez-Gómez et al. 2003).

Coeficiente de variación

En la Tabla 7, se aprecian los coeficientes de variación $(\mathrm{CV})$ obtenidos por variable dependiente en los trabajos de maestría revisados, siendo que el 27,27 por ciento de los experimentos no reportan valores de CV. En los experimentos que si reportan valores, los rangos más frecuentes de CV son 10 ? 20 y 20 ? 50 por ciento con 23,78 y 20,28 por ciento respectivamente; las frecuencias más bajas corresponden a los extremos $i^{2}$ por ciento y $i 100$ por ciento de CV.

Normalmente en los ensayos agrícolas de campo los CV se consideran bajos cuando son inferiores a 10 por ciento; medios de 10 a 20 por ciento, altos cuando van de 20 a 30 por ciento y muy altos cuando son superiores a 30 por ciento (Pimentel Gomes 1985); valores de CV por encima de 30 por ciento, indican baja precisión en el experimento y los datos deberían ser descartados (Patel et al. 2001); aunque esta medida de dispersión, puede presentar inconsistencias como indicador de variabilidad en los experimentos, por lo que no deberían considerarse del todo estos rangos de calidad (Ramiro y Caballero 2011).

Tabla 7. Coeficiente de variación obtenido por variable dependiente en los trabajos de maestría del postgrado en Agricultura Tropical, durante el periodo 1998-2016. 


\begin{tabular}{|l|l|l|}
\hline $\begin{array}{l}\text { Coeficiente de varia- } \\
\text { ción (\%) }\end{array}$ & $\mathbf{n}$ & $\mathbf{\%}$ \\
\hline Sin descripción & 312 & $\mathbf{2 7 , 2 7}$ \\
\hline$<2$ & 20 & 1,75 \\
\hline $2-5$ & 66 & 5,77 \\
\hline $5-10$ & 179 & 15,65 \\
\hline $10-20$ & 272 & $\mathbf{2 3 , 7 8}$ \\
\hline $20-50$ & 232 & $\mathbf{2 0 , 2 8}$ \\
\hline $50-100$ & 50 & 4,37 \\
\hline$>100$ & 12 & 1,05 \\
\hline$>200$ & 1 & 0,07 \\
\hline
\end{tabular}

El análisis de correlación de Spearman detectó correlación negativa altamente significativa entre el tamaño de la UE y el coeficiente de variación, lo que indica que a medida que aumenta en magnitud el tamaño de la UE disminuye el coeficiente de variación del análisis estadístico aplicado. Escobar et al. (1990) y Ramírez et al. (2013) describen tendencias de asociación similares, en pruebas paramétricas y no paramétricas, respectivamente, corroborando que a mayor tamaño de parcela o de muestra, la variación se hace más pequeña. Milton et al. (2001) señalan que en muestras de mayor tamaño, la función de distribución se aproxima a la curva de distribución normal con idéntica media y varianza inversamente proporcional al tamaño de la muestra. Puente et al. (2013) afirman que el aumento en tamaño de la muestra o número de repeticiones favorece desde el punto de vista estadístico, pero está limitado por la relación costo-beneficio en que puede incurrir el investigador.

Supuestos del ANAVA

Los supuestos del ANAVA se muestran en la Tabla 8, donde se constata que solo el 30,55 por ciento de los trabajos analizan el supuesto de normalidad de la varianza, y solo el 11,11 por ciento de los trabajos corrobora la homogeneidad de varianzas; ningún trabajo revisado consideró el supuesto de independencia.

Tabla 8. Supuestos del ANAVA considerados en los trabajos de maestría del Postgrado en Agricultura Tropical, durante el periodo 1998-2016.

\begin{tabular}{|l|l|l|}
\hline Supuestos del ANAVA & N & \% \\
\hline Normalidad & 11 & 30,55 \\
\hline Homogeneidad & 4 & 11,11 \\
\hline Independencia & 0 & 0 \\
\hline
\end{tabular}

Aunque muchas pruebas paramétricas y no paramétricas son robustas al no cumplimiento de algunos supuestos, es muy importante analizarlos y observar los efectos de su incumplimiento, su omisión puede provocar errores de interpretación, encontrar asociación entre variables cuando no existe, encontrar diferencias entre tratamientos cuando no existen o viceversa, entre otros(Montoya-Márquez et al. 2011). No todos los supuestos tienen igual importancia, la prueba ANAVA se considera robusta ante el incumplimiento de normalidad, sobre todo con mayor número 
de observaciones; sin embargo, es bastante sensible a la falta de homogeneidad e independencia (Dawson-Saunders y Trapp 1993).

La falta de independencia de los errores es bastante peligrosa y difícil de corregir, que puede anular los resultados y las posibles conclusiones de un experimento, la forma de prevenirla es cumplir el principio de aleatorización (Kuehl 2001). Ocurre mayormente al trabajar con series temporales o espaciales, para lo cual ajustan mejor los modelos de análisis intrasujeto, entre ellos, las medidas repetidas (Correa 2004).

Algunas variables, por su naturaleza, ameritan el uso de funciones matemáticas para transformar los datos y tratar que cumplan los supuestos del ANAVA (Ramiro et al. 2017); entre las funciones más utilizadas están: raíz cuadrada, para números enteros pequeños (recuentos y porcentajes); logarítmica, en números enteros positivos que cubran amplio intervalo, y angular o arcsen, recomendada para datos porcentuales que cubran un intervalo alto de valores (Steel y Torrie 1985).

Nivel de significancia

El 17,39 por ciento de los trabajos de maestría del PAT no reportan el nivel de significancia utilizado; mientras que, en los trabajos que reportan niveles de significancia, el 82,61 por ciento utilizó el nivel de 0,05 o 5 por ciento de probabilidad para interpretar efecto significativo en la prueba estadística aplicada; el 8,70 por ciento de los trabajos utilizó 0,01 o 1 por ciento, mientras que un 2,17 por ciento de los trabajos utilizó 0,1 o 10 por ciento de probabilidad para interpretar el efecto.

Un nivel de significancia estadística, entendido como alpha de la prueba, es una declaración de lo improbable que puede ser un resultado, si la hipótesis nula es verdadera para ser considerada significativa; en otras palabras, cuanto se está dispuesto a arriesgar para asumir una conclusión equivocada (Cohen 1988). Los criterios más utilizados son las probabilidades de 0,$05 ; 0,01$ y 0,001 ; el nivel de confianza de la prueba, se expresa como 1-Alpha, siendo inversa la relación entre significancia y confianza (Tosta 2013). Aunque experimentos de tamaño pequeño pueden requerir niveles superiores de significancia, hasta de 0,10 (10 por ciento), para rechazar la hipótesis nula en agricultura (Steel y Torrie 1985). Este nivel de significancia se utiliza solo en condiciones experimentales particulares (Muguiro et al. 2017), debido al inconveniente de aumentar la probabilidad, pues disminuye la confianza del procedimiento estadístico.

Interpretación de interacciones significativas

La Tabla 9 muestra las interpretaciones realizadas a la interacción significativa obtenida en los trabajos de maestría revisados, siendo la más frecuente el uso de pruebas de media generalizada sin descomposición del efecto (84,62 por ciento), lo cual resulta incorrecto según diversas investigaciones. La interpretación por curvas de regresión ocurre en 7,59 por ciento y con menor frecuencia se encuentran las pruebas de media por factor y su posterior combinación, así como la descomposición estadística de la interacción significativa (3,85 por ciento).

Tabla 9. Interpretación de la interacción significativa obtenida en los trabajos de 
grado del Postgrado en Agricultura Tropical, durante el periodo 1998-2016.

\begin{tabular}{|l|l|l|}
\hline Interpretación de la interacción & N & \% \\
\hline $\begin{array}{l}\text { Prueba de media generalizada sin descompo- } \\
\text { ner el efecto }\end{array}$ & 22 & $\mathbf{8 4 , 6 2}$ \\
\hline Descomposición de interacción significativa & 1 & 3,85 \\
\hline $\begin{array}{l}\text { No se descompone el efecto, presenta curvas } \\
\text { de regresión }\end{array}$ & 2 & 7,69 \\
\hline $\begin{array}{l}\text { Prueba de media por factor y luego se com- } \\
\text { binan }\end{array}$ & 1 & 3,85 \\
\hline
\end{tabular}

En publicaciones con experimentos factoriales, frecuentemente los efectos significativos de interacción son ignorados, analizados sin interpretación o analizados e interpretados incorrectamente (Pardo et al. 2007; Sahagún-Castellanos et al. 2008); la interacción significativa indica que los factores no son independientes entre sí, en este caso, los efectos simples de un factor dependerán del nivel del otro factor del termino de interacción (Steel y Torrie 1985), por lo que, analizar e interpretar efectos simples (por factor) resulta contradictorio e inapropiado.

En estos experimentos, los tratamientos (combinación de niveles de cada factor), bajo ningún concepto, deben ser analizados con pruebas de comparaciones múltiples (Duncan, Tukey, Scheffe), lo que implicaría ignorar la estructura factorial y renunciar a las ventajas que ella ofrece (Petersen 1977; Blanco 2001); además de fragmentar el orden lógico de los tratamientos, estas pruebas pueden añadir cuotas propias de error de interpretación, facilitando que el investigador incurra en error tipo I y genere falsas expectativas (Nelson y Rawlings 1983; Mihail y Niblack 1991).

Sahagún-Castellanos et al. (2008) señalan que el método adecuado de análisis e interpretación de la interacción significativa está condicionado por el tipo de variable independiente involucrada. Las pruebas de comparaciones múltiples serán apropiadas para factores cualitativos cuyos niveles no posean jerarquía (no estructurados), aplicadas dentro de cada nivel del segundo factor, prefiriendo aquellas que eviten el error tipo I. Para factores cuyos niveles pueden asociarse con puntos en escala numérica, el análisis estadístico más adecuado se basa en el ajuste de una función de regresión. Para factores cualitativos estructurados, la recomendación es emplear contrastes ortogonales, que pueden opacar la interacción, pero permiten interpretar de manera más sencilla y acertada el efecto de los tratamientos.

Garrido (2008) señala que la interpretación correcta de una interacción significativa exige plantear comparaciones lineales de un grado de libertad (desglosamiento), haciendo posible aislar y agotar el significado de la interacción; el objeto es detectar si un efecto simple concreto es o no el mismo en los diferentes niveles del segundo factor. Luego de desglosar el efecto, el investigador puede apoyar el análisis, según sea el caso, con pruebas complementarias de regresión o incluso con análisis gráfico.

Condiciones complementarias observadas

Como condiciones complementarias observadas en los trabajos de maestría revi- 
sados se determinó que 82,61 por ciento de los trabajos del PAT presentan los resultados por UE en el apéndice; mientras que sólo el 13,04 por ciento de los trabajos presentan resumen del ANAVA dentro de la discusión.

El apéndice del trabajo de grado, constituye todo aquello que, siendo diseñado por el autor, se deja para el final como complemento o apoyo (Orozco et al. 2013); en esta parte pueden registrarse datos relacionados directa o indirectamente con el estudio (Rodríguez 2005), incluso datos de todas las repeticiones del experimento (Fuentes 2015). En pro de la veracidad y calidad estadística de las investigaciones, la colocación de datos originales en el apéndice del trabajo, puede permitir demostrar los supuestos de parametricidad de cada experimento; aunque podría realizarse plagio de la información.

La disponibilidad de cuadros resumen del ANAVA durante la discusión de resultados del manuscrito científico, permite visualizar rápidamente los efectos del tratamiento sobre las distintas variables respuestas evaluadas, dando al lector una perspectiva general del estudio; además, para experimentos factoriales es posible visualizar la interacción significativa y su desglosamiento (Marcano et al. 2018). El cuadro resumen se construye con los grados de libertad y cuadrados medios de los factores en estudio y del error experimental, acompañados por los coeficientes de variación obtenidos en cada variable respuesta analizada.

\section{Conclusiones}

El uso de las técnicas estadísticas difiere entre las menciones del Postgrado en Agricultura Tropical.

En los trabajos de maestría se emplea mayormente diseños experimentales y arreglos de tratamientos robustos, con predominio de análisis estadísticos acordes con la condición experimental; sin embargo, predomina el uso de pruebas de comparación de medias que inducen a error tipo I.

En la mayoría de las tesis, los experimentos emplean menos de cuatro repeticiones por tratamiento; la unidad experimental, no está debidamente descrita o está representada por solo una observación.

De manera general, se ignoran los supuestos del ANAVA y su análisis previo, y de forma similar, se interpretan inadecuadamente los efectos de interacción significativa.

Recibido:19/11/2019

Aceptado:14/04/2020 


\section{Referencias}

Alegría, V. A. M. (1998), 'La educación agrícola en la universidad de oriente, venezuela', Ceiba 39(1), 161-163.

Angarita, M. A. O. \& Parra, A. B. S. (2014), 'Importancia de la probabilidad y la estadística en la formación del ingeniero', I3+ 1(2), 26-37.

Arriaza Balmon, M. (2006), 'Guía práctica de análisis de datos', IFABA, España .

Badii Zabeh, M. H., Castillo, J., Landeros, J. \& Cortez, K. (2007), 'Papel de la estadística en la investigación científica = role of statistics in scientific research.', Innovaciones de Negocios 4(7), 107-145.

Balzarini, M., Di Rienzo, J., Tablada, M., Gonzalez, L., Bruno, C., Córdoba, M., Robledo, W. \& Casanoves, F. (2012), 'Estadística y biometría', Ilustraciones del uso de Infostat en problemas de agronomía. Universidad Nacional de Córdoba .

Baray, H. L. Á. (2006), Introducción a la metodología de la investigación, Juan Carlos Martínez Coll.

Blanco, F. A. (2001), 'Métodos apropiados de análisis estadistico subsiguientes alánalisis de varianza (andeva)', Agronomía Costarricense 25(1), 53-59.

Boubeta, A. R. \& Mallou, J. V. (2014), Estadística práctica para la investigación en ciencias de la salud, Netbiblo.

Carmer, S. G. \& Swanson, M. R. (1973), 'An evaluation of ten pairwise multiple comparison procedures by monte carlo methods', Journal of the American Statistical Association 68(341), 66-74.

Catalán, P. (2015), '¿ por qué es importante investigar en botánica?', Lucas Mallada. Revista de Ciencias (17), 9-13.

Chew, V. (1976), Comparing treatment means: a compedium [analysis of experimental data]., Technical report.

Cohen, J. (2013), Statistical power analysis for the behavioral sciences, Academic press.

Correa, L. (2004), 'Análisis de medidas repetidas. 41 p', Universidad Nacional de Colombia, Medellín, Colombia .

Cortés, C. (2011), Propiedades y aplicaciones de los cuadrados latinos, Master's thesis, Universidad Autónoma Metropolitana, México.

Dawson-Saunders, B. \& Trapp, R. G. (1993), Bioestadística médica, El Manual Moderno México DF.

Comunicaciones en Estadística, junio 2020, Vol. 13, No. 1 
Di Rienzo, J., Casanoves, F., Balzarini, M., Gonzalez, L., Tablada, M., Robledo, C. et al. (2012), 'Grupo infostat, fca, universidad nacional de córdoba, argentina', URL http://www. infostat. com. ar.

Díaz Reissener, C. \& Rivas Martínez, G. (2015), 'Fundamentos para la aplicación de bioestadística en odontología (parte 2)', Rev. salud pública Parag pp. P56P61.

Echavarría Sánchez, H., Correa Londoño, G., Patiño Díez, J. F., Acosta Jaramillo, J. J., Rueda Restrepo, J. A. et al. (2006), 'Evaluación de métodos estadísticos utilizados en trabajos de grado y tesis de los programas de la facultad de ciencias agropecuarias, en un período de tres años.', Revista Facultad Nacional de Agronomía Medellín; Vol. 59, núm. 2 (2006); 3465-3580 2248-7026 03042847 .

Escobar Soto, C., Buitrago, I. D. J. \& Jaramillo, J. I. (1990), 'Tamaño de parcela experimental y número de repeticiones para ensayos de rendimiento con caupi, vigna ungiculata (l) walp', Revista Facultad Nacional de Agronomía Medellín

Estrada, C. \& Martínez, V. (2003), 'Estabilidad del rendimiento de grano de híbridos de maíz usando mejores predictores lineales insesgados', Agrociencia 37, 605-616.

Fernández, R., Trapero, A. \& Domínguez, J. (2010), 'Análisis de la varianza', Experimentación en agricultura. Seville. Spain: Consejería de Agricultura y Pesca, Servicio de Publicaciones y Divulgación pp. 978-84.

Fuentes, O. D. P. (2015), 'Guía metodológica'.

Fuentes, R. et al. (2015), 'Análisis de variables múltiples', Revista Chilena de Anestesia pp. 106-11.

Garrido García, J. (2008), 'La interacción entre factores en el análisis de varianza: errores de interpretación'.

Gómez-Gómez, M., Danglot-Banck, C. \& Vega-Franco, L. (2003), 'Sinopsis de pruebas estadísticas no paramétricas. cuándo usarlas', Revista Mexicana de Pediatría 70(2), 91-99.

González, M. I. O., Orozco, A. P., Asorey, C. P. \& García, L. I. R. (2013), 'El abc en la revisión de una tesis de grado', MediSan 17(12), 9180-9192.

Jiménez Valderrama, M. T. P. \& Reyes Villamil, M. A. (2013), 'Evolución de los desarrollos estadísticos en la agronomía', Revista de la Universidad de la Salle 2013(62), 307-321.

Johnson, D. E. (2000), Métodos multivariados aplicados al análisis de datos, number 519.535 J66m, México, MX: International Thomson Ed.

Johnson, S., SB, J. \& RD, B. (1982), 'On the status of statistics in phytopathology'. 
Kirk, R. E. (1995), 'Experimental design: procedures for the behavioral sciences. brooks', Cole, Pacific Grove, CA .

Kuehl, R. O. (2001), Diseño de experimentos: Principios estadísticos de diseño y análisis de investigación.

Lizarzaburu Montero, L. M. (2013), 'Uso de la estadística en trabajos de investigación en la universidad san pedro'.

Madden, L., Knoke, J. \& Louie, R. (1982), 'Considerations for the use of multiple comparison procedures in phytopathological investigations', Phytopathology 72, 1015-1017.

Maindonald, J. \& Cox, N. (1984), 'Use of statistical evidence in some recent issues of dsir agricultural journals', New Zealand journal of agricultural research 27(4), 597-610.

Marcano, G. R., Acuña, R. S. \& Cuevas, M. S. (2018), 'Tipos de sustrato y frecuencias de riego sobre características agronómicas de la moringa (moringa oleifera lamark) cv. super genius en condiciones de vivero - types of substrate and irrigation frequencies on the agronomic characteristics of moringa (moringa oleifera lamark) cv. super genius in nursery conditions', SABER 30, 228-237.

Martínez, O. \& Galindo, R. (1994), 'Una revisión analítica de diseños y métodos estadísticos en ciencias agrícolas', Agronomía Colombiana 11(1), 90-94.

Mihail, J. \& Niblack, T. (1991), 'Comparison of treatment means: A statistical fantasy', Journal of nematology $\mathbf{2 3}(4 \mathrm{~S}), 557$.

Milton, J. S. (2001), Estadística para biología y ciencias de la salud, number QH 323.5. M5418 2001.

Montgomery, D. C. (2003), Diseño y análisis de experimentos, Limusa-Wiley.

Montoya-Márquez, J. A., Sánchez-Estudillo, L. \& Torres-Hernández, P. (2011), 'Diseños experimentales ¿ qué son y cómo se utilizan en las ciencias acuáticas?', Ciencia y mar 15(43), 61-70.

Monzón, D. (1992), Introducción al diseño de experimentos, Universidad Central de Venezuela, Facultad de Agronomía.

Muguiro, A., Alvarez, C., Babinec, F. J. \& Marano, R. P. (2017), Rehabilitacion de suelos hortícolas: impacto de prácticas agrícolas con uso de yeso y drenaje= soils with physical impediments and horticultural crops: impact of agricultural management with gypsum and drainage, Technical report, Universidad Nacional del Litoral. Facultad de Ciencias Agrarias.

Nelson, L. A. \& Rawlings, J. O. (1983), 'Ten common misuses of statistics in agronomic research and reporting', Journal of Agronomic Education 12(1), 100105. 
Orlandoni, G. (2010), 'Escalas de medición en estadística', Telos 12(2), 243-243.

Pardo, A., Garrido, J., Ruiz, M. Á. \& San Martín, R. (2007), 'La interacción entre factores en el análisis de varianza: errores de interpretación', Psicothema 19(2), 343-349.

Patel, J. K. (2001), Coefficient of variation in field experiments and yardstick thereof-an empirical study, $\mathrm{PhD}$ thesis, AAU, Anand.

Petersen, R. (1977), 'Use and misuse of multiple comparison procedures 1', Agronomy Journal 69(2), 205-208.

Pimentel-Gomes, F. (1985), 'Curso de estatística experimental 11. ed', São Paulo, Nobel .

Puente, H. F., Bertot, R. R., Cárdenas, V. T., Navarro, M. V. \& Villafranca, M. H. (2013), 'El estudio de la variabilidad en la producción de huevos de gallinas white leghorn alimentadas con hcp', REDVET. Revista Electrónica de Veterinaria 14(9), 1-10.

Ramírez, I. C., Barrera, C. J. \& Correa, J. C. (2013), 'Efecto del tamaño de muestra y el número de réplicas bootstrap', Ingeniería y Competitividad 15(1), 93-101.

Ramiro Vásquez, E., Caballero Núñez, A. \& Herrera Villafranca, M. (2017), 'Transformación de variables binomiales para su análisis según un diseño de bloques al azar', Cultivos Tropicales 38(1), 108-114.

Raudonius, S. (2017), 'Application of statistics in plant and crop research: important issues.', Zemdirbyste-Agriculture 104(4).

Rendón-Macías, M. E., Villasís-Keeve, M. Á. \& Miranda-Novales, M. G. (2016), 'Estadística descriptiva', Revista Alergia México 63(4), 397-407.

Rodríguez Moguel, E. A. (2005), 'Metodología de la investigación', México: Universidad Juarez autónoma de tabasco .

Ruiz-Ramírez, J., Pérez-Salazar, C., Cruz-Kuri, L. \& Hernández-Rodríguez, G. E. (2012), 'Programa que calcula la eficiencia relativa de los diseños experimentales', Terra Latinoamericana 30(1), 97-100.

Sabadías, A. V. (1995), Estadística descriptiva e inferencial, Vol. 8, Univ de Castilla La Mancha.

Sahagún-Castellanos, J., Martínez-Garza, A. \& Rodríguez-Pérez, J. (2008), 'Problemas y métodos comunes del análisis de experimentos factoriales', Revista Chapingo. Serie horticultura 14(2), 213-222.

Silva-Acuña, R., Álvarez, V. H. \& Silva-Acuña, A. (2000), 'Como comparar correctamente tratamientos de naturaliza cualitativa', Agronomía Tropical $\mathbf{5 0}(2), 151-155$. 
Sousa, V., Driessnack, M. \& Costa, I. (2007), 'Revisión de diseños de investigación resaltantes para enfermería. parte 1: Diseños de investigación cuantitativa', Rev latino-am enfermagem 15(3), 1-6.

Steel, G. \& Torrie, J. (1985), 'Bioestadística: Principios y procedimientos (2da. ed.) mcgraw-hill', México, $D F$.

Tabachnick, B. G. \& Fidell, L. S. (2001), 'Using multivariate statistics. allyn and bacon', Needham Heights, MA .

Thiese, M. S., Arnold, Z. C. \& Walker, S. D. (2015), 'The misuse and abuse of statistics in biomedical research', Biochemia medica: Biochemia medica $\mathbf{2 5}(1), 5-11$.

Tosta, M. (2013), 'El poder estadístico. diferencias observadas cuando se cambia el alfa establecido en un estudio de investigación', Scientific International Journal 10(1), 23-32.

Universidad de Oriente (UDO) (2015), Postgrado en Agricultura Tropical., Universidad de Oriente (UDO), Venezuela.

*http://www.udo.edu.ve/index.php/component/flexicontent/23-

postgrado/89-postgrado-en-agricultura-tropical

Vásquez, E. R. \& Núñez, A. C. (2011), 'Inconsistencia del coeficiente de variación para expresar la variabilidad de un experimento en un modelo de análisis de varianza', Cultivos Tropicales 32(3), 59-62.

Yan, W., Cornelius, P. L., Crossa, J. \& Hunt, L. (2001), 'Two types of gge biplots for analyzing multi-environment trial data', Crop Science 41(3), 656-663. 